\title{
Evaluation of Fetal Distress Diagnosis during Delivery Stages based on Linear and Nonlinear Features of Fetal Heart Rate for Neural Network Community
}

\author{
Zafer Cömert \\ Bitlis Eren University, \\ Department of Computer Engineering
}

\author{
Adnan Fatih Kocamaz \\ İnönü University, \\ Department of Computer Engineering
}

\begin{abstract}
Cardiotocography (CTG) is a fetal monitoring technique used to determine the distress level of the fetus during pregnancy and delivery. CTG consists of two different signals including fetal heart rate (FHR) and uterine contraction (UC) activities. The linear features of FHR are the most powerful prognostic indices to ascertain whether the fetus in distress. In addition, it is observed that nonlinear features have produced very great results on the time series analysis in recently. In this context, the classification success of the neural network community designed based on the linear and nonlinear features of FHR is analyzed for the delivery process evaluated in three stages. The experimental results have shown that the system designed to distinguish normal and pathological instances is achieved the best classification accuracy at the first stage of the analysis. Also, the greatest contribution of nonlinear features to the classification accuracy is observed at the second stage of the delivery. Consequently, $92.40 \%, 83.29 \%$, and $79.22 \%$ of accuracy obtained for the three stage of the analysis, respectively.
\end{abstract}

\section{General Terms}

Electronic fetal monitoring, cardiotocography, fetal heart rate, neural networks

\section{Keywords}

Cardiotocography, fetal heart rate, artificial neural network, linear and nonlinear features, classification

\section{INTRODUCTION}

Cardiotocography (CTG) is a monitoring technique used widely over all the world in order to determine the distress level of the fetus. CTG comprises of two main signals which are fetal heart rate (FHR) and uterine contraction (UC) recorded as simultaneously [1]. Figure 1 illustrates a sample digitized CTG record. The greatest advantage of using CTG is that it contributes to the early diagnosing of undesirable events, such as cerebral palsy and intrapartum fetal hypoxia developing depend on the lacking of oxygen. Regrettably, it observed that the rates of cerebral palsy have remained stable so far, and the rates of caesarean section and instrumental delivery have increased depending on the use of CTG [2]. In clinical practice, the interpretation of CTG is performed subjectively by a visual inspection way. Observes (gynecologist, nurse or midwife) try to catch some specific patterns of FHR known as morphological features with a naked eye. The workload of observers, their expertise levels, and emotional situations prevent an objective assessment. Moreover, CTG has a low specificity or has a high false
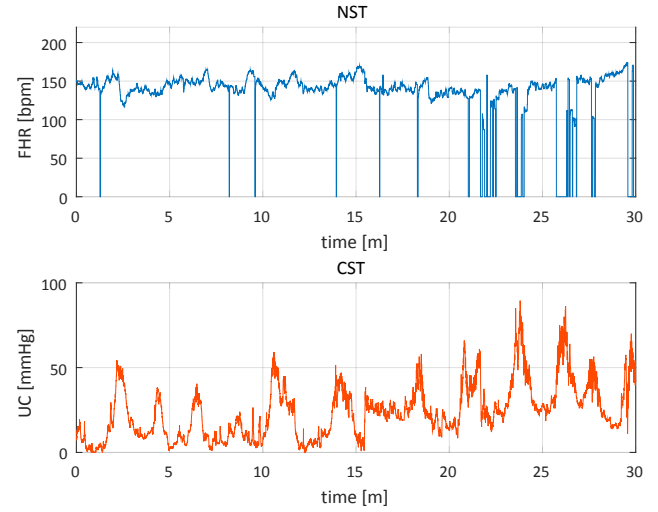

Figure 1. A digital CTG record

positive rate [3]. This situation leads to poor judgment. The many records that must be evaluated as normal are labeled as pathological, and actually, the real pathological records may be overlooked. Also, it has also been reported that there is a high level of disagreement between inter- and intra-observed in the interpretation of CTG [4]. The computer-aided automatic FHR analysis is seen as the most promising method to overcome these problems [5]. Several guidelines have been published by different health organizations to ensure a consistent interpretation of CTG [5-7]. These guidelines focus mainly on the shapes and changes in FHR. Naturally, the first computer-aided studies have focused on the automatic detection of some specific patterns like baseline heart rate, variability, acceleration and deceleration patterns that are specified in the guidelines as morphological features [8]. Then, the spectral analysis of heart rate variability (HRV) has yielded very satisfactory results [9]. In recent studies, it is noticed that nonlinear signal analysis has increased the diagnostic power of HRV $[8,10,11]$. Entropy estimators, complexity indices, such as Lempel-Ziv [12], and other nonlinear parameters are used to obtain a more information about FHR and to provide a better representation of the signals.

In this proposal, the classification success of an artificial neural network (ANN) community for the delivery process evaluated in the three stages is investigated based on linear and nonlinear features of FHR. The rest of paper is organized as follow. Section 2 covers the summary of the data collection and methods. The experimental results and a final discussion are given in Section 3. Finally, concluding remarks are given in Section 4. 


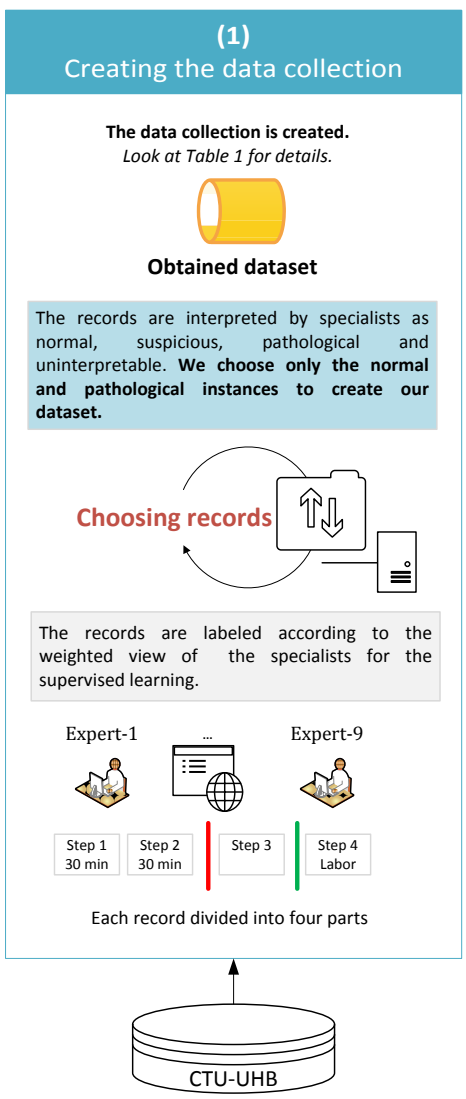

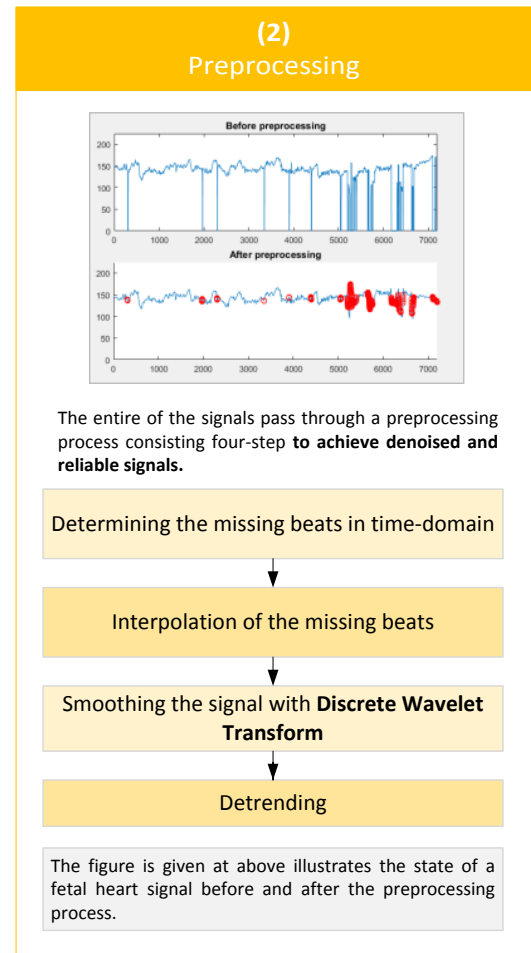

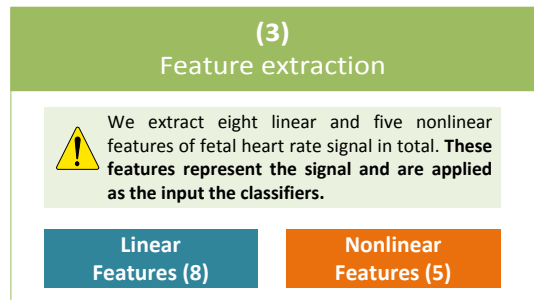

Look at Table 2 for the detail description of the feature

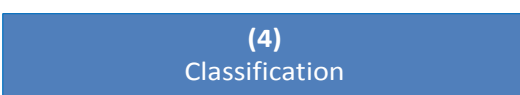

An artificial neural network with different trainin algorithms employ to classify the fetal heart rat signals. The network topology is set as $\{13,[10,3], 2\}$.

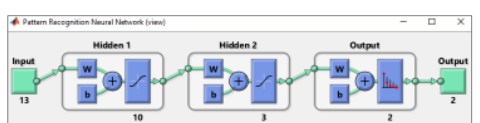

10 -fold cross-validation is used to achieve more reliable results.

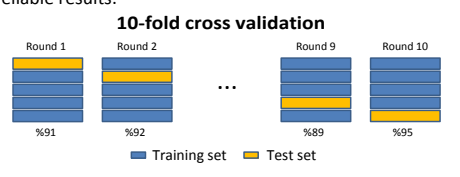

- The analysis is separately conducted on the delivery process evaluated in three stages.

- The subsets consisting linear, nonlinear, and the combination of them are applied as the input to the an ANN classifier. Table 4 - Table 6 can be review for the comparison of results.

Figure 2. The block diagram of the analysis

\section{MATERIAL AND METHOD}

In this proposal, an open-access database named CTU-UHB [13] has been used. Figure 2 illustrates the block diagram of the analysis and important tips. The entire of the signals in the database have been passed through a four-step preprocessing process briefly described in our previous work [14]. Linear and nonlinear indicators of FHR have been revealed in the feature extraction stage. At the final stage, these features have been applied as the input to ANN.

\subsection{Data Collection}

CTU-UHB database consists of a total of 552 raw CTG records. The each record in the database is divided into four parts and evaluated by nine experienced specialists. The first two parts of the records represent the first stage of the delivery, and the third part represents the second stage of delivery. These stages of analysis are symbolized as I, II, and III, respectively. The first three stages of the records were labeled as normal $(\mathrm{N})$, suspicious $(\mathrm{S})$, pathological $(\mathrm{P})$, and uninterpretable (U). Similarly, the last part of the records is labeled as no hypoxia, mild hypoxia, severe hypoxia, and uninterpretable depending on the parameters obtained after the delivery, such as pH, Apgar score, and birth weight.

CTG records must be labeled before the analysis in terms of the supervised learning. Two fundamental approaches are used for this purpose. In the first approach, clustering techniques, such as fuzzy or neuro-fuzzy [15] are used on the neonatal outcomes, and a retrospective evaluation is conducted. In the second approach, specialist views is considered [16]. As stated earlier, the disagreement level on the interpretation of CTG between inter- and even intraobservers is high. For this reason, the views of all specialists have been taken into consideration separately for all stages of the analysis, and the required labeling was made according to the weighted view of the specialists. Also, uninterpretable and suspicious records have been isolated from the dataset to provide a reliable binary classification. Table 1 gives the distribution of the records during all stages of the analysis.

Table 1. The distribution of the records during the analysis

\begin{tabular}{|c|c|c|c|c|}
\hline Analysis steps & N & S & P & U \\
\hline I & 272 & 230 & 44 & 6 \\
\hline II & 195 & 267 & 86 & 4 \\
\hline III & 106 & 160 & 71 & 215 \\
\hline
\end{tabular}

\subsection{Linear Features}

Traditionally, the linear features have been agreed as the primary indicators to enable evaluation of FHR signals [5]. Here, $x(n)$ denotes an FHR signal and is defined as $n=$ $1,2, \ldots, N$. The linear features are formulated as follows.

$$
\begin{aligned}
& F H R_{\text {mean }}=\bar{x}=\frac{1}{N} \sum_{i=1}^{N} x(i) \\
& F H R_{\text {std }}=\sqrt{\frac{1}{N-1} \sum_{i=1}^{N}(x(i)-\bar{x})^{2}}
\end{aligned}
$$




$$
\begin{aligned}
& L T I=I Q R\left(\left\{y(i): y(i)=\sqrt{x^{2}(i)+x^{2}(i+1)}\right\}\right) \\
& \Delta=\frac{1}{M} \sum_{i=1}^{M}\left[\max _{i \in M}(x(i))-\min _{i \in M}(x(i))\right] \\
& S T V=\frac{1}{24 M} \sum_{i=1}^{24 M}|\operatorname{sm}(i+1)-\operatorname{sm}(i)| \\
& I I=\frac{S T V}{\operatorname{std}[\operatorname{sm}(i)]}
\end{aligned}
$$

$F H R_{\text {mean }}$ represents the mean value of FHR, and $F H R_{\text {std }}$ expresses the standard deviation of FHR. The long-term irregularity is indicated by LTI. The delta $(\Delta)$ considers the variability for each minute, and $M$ depicts the total number of minutes. $\mathrm{sm}$ denotes a vector and is obtained by collecting one sample per 2.5 seconds from FHR signals. The short-term variability is expressed as STV and is calculated based on the creation interval of $\mathrm{sm}$ vector. The interval index is symbolized by II and shows the gross change in the FHR. In addition to these features, the rates of the number of acceleration and deceleration patterns of the signal length were also considered.

\subsection{Nonlinear features}

Nonlinear features are an efficient approach to extract the characteristic attributes of FHR signals unobservable in the time-domain analysis [17]. Nonlinear analysis is based on the models developed to ascertain the complex phenomena in essence. In this context, there are already several methods for the analysis of nonlinear time series [10]. The present proposal has employed the Approximate Entropy (ApEn), Sample Entropy (SampEn), and Lempel-Ziv Complexity $(L Z C)$.

Entropy defines the behavior of a system in terms of randomness and measures the underlying dynamic knowledge of the system. $\operatorname{ApEn}(m, r)$ can separate the low-dimensional deterministic systems, chaotic systems, stochastic and mixed systems [18]. A time series of $N$-length and shown with $x_{n}$ is divided by a set of $m$-length vectors denoted by $u_{m}(i)$. In the next step, the $u_{m}(i)$ and $u_{m}(j)$ vectors are expressed a number called as $n_{i}^{m}(r)$ in terms of Euclidean sense $d\left[u_{m}(i), u_{m}(j)\right] \leq r$. The number is used to calculate the probability of vectors to being close according to $C_{i}^{m}(r)=$ $n_{i}^{n} /(N-m+1)$. Finally, $A p E n$ is defined as follows.

$$
\begin{aligned}
\operatorname{ApEn}(m, r, N)= & \frac{1}{N-m+1} \sum_{i=1}^{N-m+1} \ln \left(C_{r}^{m}(i)\right) \\
& -\frac{1}{N-m} \sum_{i=1}^{N-m} \ln \left(C_{r}^{m+1}(i)\right)
\end{aligned}
$$

Herein, the Equation 7 can be written in compressed form as $\operatorname{ApEn}(m, r)=\lim _{N \rightarrow \infty}\left[\phi^{m}(r)-\phi^{m+1}(r)\right]$. Herein, $r$ acts as a tolerance (threshold) value and is used as $r=(0.15 ; 0.2)$ and embedding dimension set as $m=2$ in this study.

$S a m p E n$ is calculated very similarly to $A p E n$ with very small modifications made on ApEn [19]. Unlike ApEn, SampEn does not count the self-matches. When compared to $A p E n$, it reduces the biases. SampEn is defined as follow. The $m$ and $r$ parameter values are the same as for $A p E n$.

$\operatorname{SampEn}(m, r)=\lim _{N \rightarrow \infty}-\ln \frac{C^{m+1}(r)}{C^{m}(r)}$

LZC estimates the repeating patterns in time-series irrespective of time. A periodic signal has the same repeating pattern and has a low complexity. On the other hand, nonstationary signals have rarely reoccurring pattern and have the high complexity values [12].

A new sequence $(S)$ denoted as $x(1), x(2), \ldots, x(n)$ is defined to express the signal in a new form. In the case of an increase in $S, x(i+1)>x(i)$, the active index is set as 1 , in other cases, $x(i+1) \leq x(i)$, the active index is encoded by 0 . The algorithm counts the same patterns in $S$. The complexity is increased by 1 for each new pattern. When the last element of the $S$ is arrived, $c(n)$ is increased by 1 , traditionally. $c(n)$ depends on the length of the original signal $(n)$. The normalization is performed as described below to remove this dependency.

$C(n)=\frac{\mathrm{c}(\mathrm{n}) \log _{2}(n)}{n}$

\subsection{Artificial Neural Network}

Artificial neural network (ANN) is a computational technique inspired by the learning and generalization ability of the human brain. ANN is used for many purposes, such as function convergence, pattern recognition, and classification in many fields of science [20]. From a technical point of view, ANN consists of an input layer, one or more hidden layer(s), and an output layer [21]. In addition, various types of ANN are associated with learning rules and is based on the solution of a series optimization problems. A neuron output representing $\mathrm{i}^{\text {th }}$ layer $\left(y^{i}\right)$ is calculated as follow for a multilayered architecture.

$y^{i}=\sigma\left(\sum_{j=1}^{N} w_{i j} x_{j}+b^{i}\right)$ 
where $\sigma$ is activation function, $N$ is the number of input nodes, $w_{i j}$ is the weight, $x_{j}$ is the input, and $b^{i}$ is the bias value for corresponding layer.

\section{RESULTS AND DISCUSSION}

In this study, the analysis based on a neural network community was performed separately for the delivery process evaluated in three steps. The feature set obtained as a result of the analysis is described in Table 2 . The feature set consisting of with eight linear, and five nonlinear features were reflected in two-dimensional principal component analysis (PCA) [22] space for each stage of the analysis as shown in Figure 3. The normal and pathological instances were emphasized by blue and red color in the figure, respectively.

Confusion matrix was used to measure the classification performances of neural networks having different topologies. It has four prognostic indices: True Positive (TP), True Negative (TN), False Positive (FP), and False Negative (FN). Also, confusion matrix establishes the relationship between the actual values and predicted values by the classifiers and allows the calculation of various performance criteria [23]. In this context, the performance criteria given with their mathematical forms in Table 3 were taken into account. Accuracy (Acc) signifies the total efficiency of the classifier. Sensitivity (Se) embodies the yield of the classifier on positively labeled data, and similarly, specificity $(\mathrm{Sp})$ denotes the yield of the classifier on negatively labeled data. Quality index (QI) symbolizes the geometric mean of the Se and Sp.

It is recommended to use k-fold cross-validation to achieve more reliable and accurate results on behalf of the classifier over small datasets [16]. For this reason, 10-fold crossvalidation method was used during the classification stage.

Over the network configuration, Conjugate Gradient Backpropagation with Fletcher-Reeves Restarts training algorithm was preferred because of its low memory requirement and fast convergence quality for the network training. Apart from this, Gradient descent with momentum backpropagation, Resilient Backpropagation, and LevenbergMarquardt backpropagation training algorithms were tried in the experimental work. When configuring the network, many different topologies have been used, and it has been decided to use two hidden layers consisting of ten and three hidden nodes, respectively.

The linear and nonlinear features were applied as the input to the neural network community in three different ways, separately and together. The feature set was divided into subsets as linear, nonlinear and a combination of them. The performance of the classifier was given according to the used subsets between Table 4 and Table 6. According to experimental results, the highest classification accuracy was achieved by using the combination of the linear and nonlinear features at the first step of the analysis. The classification successes that were obtained by using the relevant subsets were achieved as $92.40 \%, 83.29 \%$, and $79.22 \%$ for the delivery process evaluated in three stages.

The nonlinear features showed the greatest variability in the second stage of the delivery when FHR signal became much more irregular. As a consequence of this situation, the use of nonlinear features mostly influenced the classification accuracy in the third step of the analysis. The accuracy of $72.91 \%$ obtained by using only the linear subset increased to $79.22 \%$ as a result of using the combination of the linear and nonlinear subset.
Table 2. Description of the feature set

\begin{tabular}{lll}
\hline Symbol & Explanation & \\
\hline$F H R_{\text {mean }}$ & Average heart rate & \\
$F H R_{\text {std }}$ & Standard deviation of FHR & \\
LTI & Long-term irregularity & \\
$\Delta$ & Delta & \\
STV & Short-term variability & \\
$I I$ & Interval Index & \\
$A C C$ & The number of ACC patterns \\
& according to signal length & \\
$D C C$ & The number of DCC patterns \\
& according to signal length & \\
$A p E n(2,0.15)$ & Approximate entropy & \\
$A p E n(2,0.2)$ & Approximate entropy & \\
$\operatorname{SampEn}(2,0.15)$ & Sample entropy & \\
$\operatorname{SampEn}(2,0.2)$ & Sample entropy & \\
$\operatorname{LZC}$ & Lempel-Ziv Complexity & \\
\hline
\end{tabular}

Table 3. The performance criteria for ANN classifier

\begin{tabular}{lc}
\hline Criterion & Formula \\
\hline Acc & $\boldsymbol{T P + T N}$ \\
\cline { 2 - 2 } $\mathrm{Se}$ & $\frac{\boldsymbol{T P}+\boldsymbol{T N}+\boldsymbol{T P}+\boldsymbol{F N}}{\boldsymbol{T P}+\boldsymbol{F N}}$ \\
$\mathrm{Sp}$ & $\frac{\boldsymbol{T N}}{\boldsymbol{T N}+\boldsymbol{F P}}$ \\
$\mathrm{QI}$ & $\sqrt{\boldsymbol{S e} * \boldsymbol{S P}}$ \\
\hline
\end{tabular}

Table 4. The results obtained by using only the linear features

\begin{tabular}{ccccc}
\hline Analysis & Acc & Se & Sp & QI \\
\hline I & $\mathbf{9 1 . 7 3}$ & 94.58 & 71.83 & 81.14 \\
II & 82.56 & 87.17 & 72.34 & 79.10 \\
III & 72.91 & 77.70 & 69.17 & 72.65 \\
\hline
\end{tabular}

Table 5. The results obtained by using only the nonlinear features

\begin{tabular}{ccccc}
\hline Analysis & Acc & Se & Sp & QI \\
\hline I & $\mathbf{8 7 . 6 1}$ & 92.40 & 59.68 & 65.34 \\
II & 71.82 & 79.14 & 54.06 & 63.73 \\
III & 55.23 & 62.10 & 45.65 & 52.35 \\
\hline
\end{tabular}

Table 6. The results obtained by using the linear and nonlinear features together

\begin{tabular}{ccccc}
\hline Analysis & Acc & Se & Sp & QI \\
\hline I & $\mathbf{9 2 . 4 0}$ & 95.89 & 74.75 & 83.66 \\
II & 83.29 & 87.06 & 75.90 & 80.92 \\
III & 79.22 & 85.87 & 72.73 & 77.91 \\
\hline
\end{tabular}

Gonçalves et al. $[8,11]$ also considered the changes in the spectral field of the FHR signals while analyzing the linear and nonlinear features, and reported that there was a significant increase in the linear frequency indices immediately before delivery. Spilka et al. [10] have been able to classify FHR signals using nonlinear features, and reported 


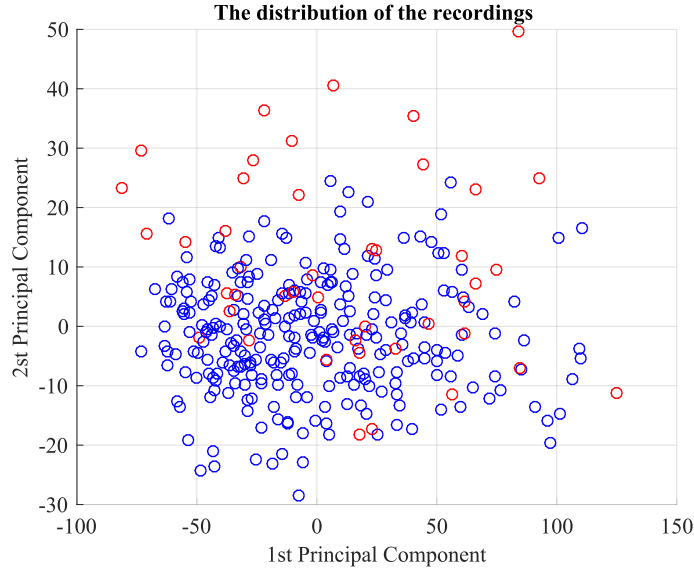

(a) The first stage of delivery, step 1 of the analysis (before maternal pushing)\

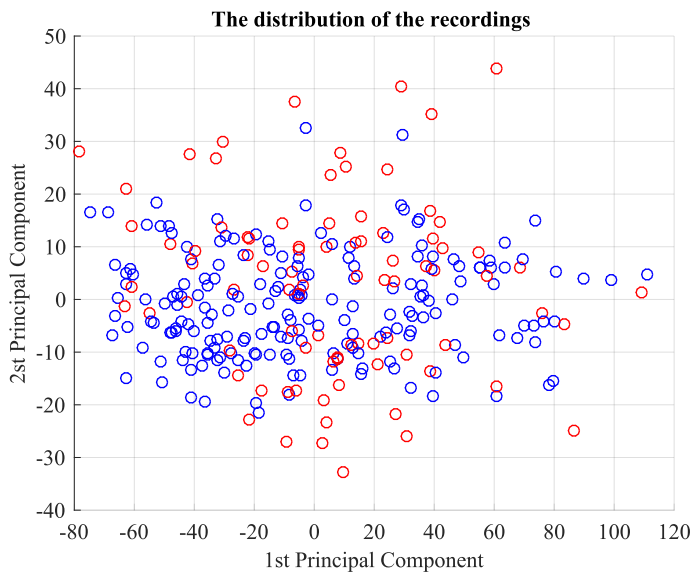

(b) The first stage of delivery, step 2 of the analysis (before maternal pushing)

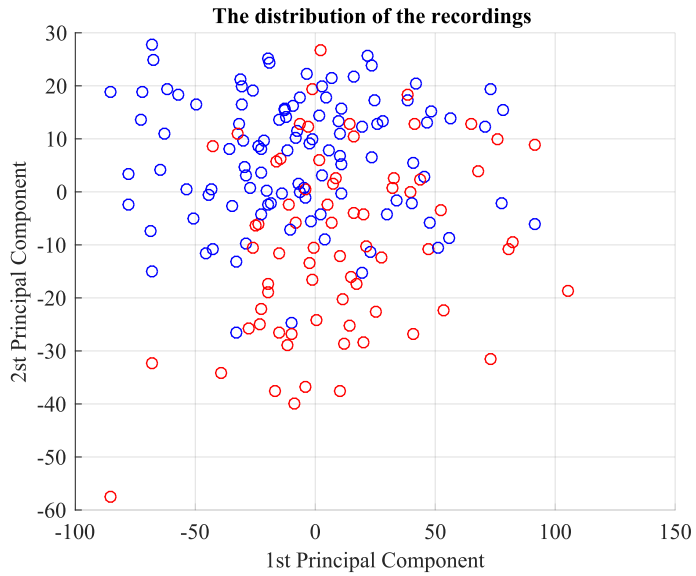

(c) The second stage of delivery, step 3 of the analysis (during maternal pushing)

Figure 3. The distribution of the records as normal and pathological throughout the delivery process on PCA space

that LZC and SampEn are the best nonlinear markers. Signorini et al. [17] have emphasized that only the use of time-domain parameters during the FHR analysis has a low estimation power. For this reason, they have stated that the use of nonlinear parameters will produce more consistent results. Logier et al. [24] has recommended the use of a new marker by defining an index on the high frequency of FHR variability. According to the research, the proposed index value of the fetuses with a high distress level is significantly lower than normal fetuses. Based on previous works published on FHR analysis, it has been said that in addition to the traditionally used linear features, the use of nonlinear features and new indices has increased the classification accuracy. Also, it is clearly seen that the use of these new indices has positively influenced the representation of CTG data and creation of the feature sets.

\section{CONCLUSION}

In this proposal, fetal distress diagnosis based on the linear and nonlinear features of FHR signals were assessed during the delivery by using an ANN community. The accuracy values of $92.40 \%, 83.29 \%$, and $79.22 \%$ were obtained for the delivery process evaluated in three stages. The highest classification accuracy was achieved by the combination of the linear and nonlinear FHR features at the first stage of the analysis. The greatest contribution of the nonlinear features on classification accuracy was observed in the second stage of the delivery, and it is seen that the irregularity is at the highest level on FHR in this process. Also, other significant contributions of the nonlinear features are observable on all performance criteria.

We restricted this work with using only the linear and nonlinear features of FHR. It clear that the nonlinear features provided more efficient representation for FHR signals. In the future design, we will focus on the supplementary features that will improve the representation power of the signals. Also, future works will advance by using time-frequency features based on different empirical bandwidths, and various machine learning techniques.

\section{ACKNOWLEDGE}

We thank Dr. Jiri Spilka due to his contribution to the coding of the Lempel Ziv Complexity.

\section{CONFLICT OF INTERESTS}

The authors declare that there is no conflict of interests regarding the publication of this paper.

\section{REFERENCES}

[1] V. Doria, A. T. Papageorghiou, A. Gustafsson, A. Ugwumadu, K. Farrer, and S. Arulkumaran, "Review of the first 1502 cases of ECG-ST waveform analysis during labour in a teaching hospital," Bjog, vol. 114, pp. 1202-7, Oct 2007.

[2] A. Pinas and E. Chandraharan, "Continuous cardiotocography during labour: Analysis, classification and management," Best Practice \& Research Clinical Obstetrics \& Gynaecology, vol. 30, pp. 33-47, 1// 2016.

[3] R. Liston, D. Sawchuck, and D. Young, "Fetal health surveillance: antepartum and intrapartum consensus guideline," J Obstet Gynaecol Can, vol. 29, pp. S3-56, Sep 2007.

[4] J. Bernardes, A. Costa-Pereira, D. Ayres-de-Campos, H. P. Geijn, and L. Pereira-Leite, "Evaluation of interobserver agreement of cardiotocograms," Int $J$ Gynaecol Obstet, vol. 57, 1997.

[5] D. Ayres-de-Campos, C. Y. Spong, E. Chandraharan, and F. I. F. M. E. C. Panel, "FIGO consensus guidelines 
on intrapartum fetal monitoring: Cardiotocography," Int J Gynaecol Obstet, vol. 131, pp. 13-24, Oct 2015.

[6] R. O. Bonow, B. A. Carabello, K. Chatterjee, A. C. De Leon Jr, D. P. Faxon, M. D. Freed, et al., "ACC/AHA 2006 guidelines for the management of patients with valvular heart disease: A report of the American College of Cardiology/American Heart Association Task Force on Practice Guidelines (Writing Committee to Revise the 1998 Guidelines for the Management of Patients with Valvular Heart Disease) - Developed in collaboration with the Society of Cardiovascular Anesthesiologists," Circulation, vol. 114, pp. e84-e231, 2006.

[7] G. A. MacOnes, G. D. V. Hankins, C. Y. Spong, J. Hauth, and T. Moore, "The 2008 National Institute of Child Health and Human Development workshop report on electronic fetal monitoring: Update on definitions, interpretation, and research guidelines," Obstetrics and Gynecology, vol. 112, pp. 661-666, 2008.

[8] H. Gonçalves, A. P. Rocha, D. Ayres-de-Campos, and J. Bernardes, "Linear and nonlinear fetal heart rate analysis of normal and acidemic fetuses in the minutes preceding delivery," Medical and Biological Engineering and Computing, vol. 44, pp. 847-855, 2006.

[9] M. Malik, A. J. Camm, J. T. Bigger, G. Breithardt, S. Cerutti, R. J. Cohen, et al., "Heart rate variability: standards of measurement, physiological interpretation and clinical use. Task Force of the European Society of Cardiology and the North American Society of Pacing and Electrophysiology," Circulation, vol. 93, pp. 104365, Mar 11996.

[10] J. Spilka, V. Chudáček, M. Koucký, L. Lhotská, M. Huptych, P. Janků, et al., "Using nonlinear features for fetal heart rate classification," Biomedical Signal Processing and Control, vol. 7, pp. 350-357, 7// 2012.

[11] H. Gonçalves, J. Bernardes, A. Paula Rocha, and D. Ayres-de-Campos, "Linear and nonlinear analysis of heart rate patterns associated with fetal behavioral states in the antepartum period," Early Human Development, vol. 83, pp. 585-591, 9// 2007.

[12] A. Lempel and J. Ziv, "On the Complexity of Finite Sequences," IEEE Transactions on Information Theory, vol. 22, pp. 75-81, 1976.

[13] V. Chudáček, J. Spilka, M. Burša, P. Janků, L. Hruban, M. Huptych, et al., "Open access intrapartum CTG database," BMC Pregnancy and Childbirth, vol. 14, pp. 16-16, 01/13 2014.

[14] Z. Cömert and A. F. Kocamaz, "A Study Based on Gray Level Co-Occurrence Matrix and Neural Network Community for Determination of Hypoxic Fetuses," presented at the International Artificial Intelligence and
Data Processing Symposium (IDAP'16), Malatya, Turkey, 2016.

[15] R. Czabanski, M. Jezewski, K. Horoba, J. Jezewski, and J. Leski, "Fuzzy Analysis of Delivery Outcome Attributes for Improving the Automated Fetal State Assessment," Applied Artificial Intelligence, vol. 30, pp. 556-571, 2016/07/02 2016.

[16] H. Sahin and A. Subasi, "Classification of the cardiotocogram data for anticipation of fetal risks using machine learning techniques," Applied Soft Computing, vol. 33, pp. 231-238, 8// 2015 .

[17] M. G. Signorini, G. Magenes, S. Cerutti, and D. Arduini, "Linear and nonlinear parameters for the analysisof fetal heart rate signal from cardiotocographic recordings," IEEE Transactions on Biomedical Engineering, vol. 50, pp. 365-374, 2003.

[18] S. M. Pincus, "Approximate entropy as a measure of system complexity," Proceedings of the National Academy of Sciences of the United States of America, vol. 88, pp. 2297-2301, 1991.

[19] J. S. Richman and J. R. Moorman, "Physiological timeseries analysis using approximate entropy and sample entropy," Am J Physiol Heart Circ Physiol, vol. 278, pp. H2039-49, Jun 2000.

[20] Z. Cömert, A. F. Kocamaz, and S. Güngör, "Cardiotocography signals with artificial neural network and extreme learning machine," in 2016 24th Signal Processing and Communication Application Conference (SIU), 2016, pp. 1493-1496.

[21] I. A. Basheer and M. Hajmeer, "Artificial neural networks: fundamentals, computing, design, and application," Journal of Microbiological Methods, vol. 43, pp. 3-31, 12/1/2000

[22] Z. Cömert and A. F. Kocamaz, "Determination of QT interval on synthetic electrocardiogram," in 2015 23nd Signal Processing and Communications Applications Conference (SIU), 2015, pp. 2569-2572.

[23] D. M. Powers, "Evaluation: from precision, recall and Fmeasure to ROC, informedness, markedness and correlation," Journal of Machine Learning Technologies, vol. 2, pp. 37-63, 20112011.

[24] R. Logier, J. D. Jonckheere, M. Jeanne, and R. Matis, "Fetal distress diagnosis using heart rate variability analysis: Design of a High Frequency Variability Index," in 2008 30th Annual International Conference of the IEEE Engineering in Medicine and Biology Society, 2008, pp. 4728-4731. 